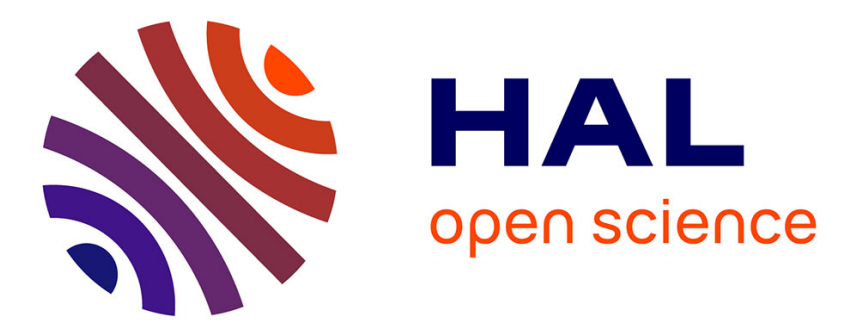

\title{
Power-Efficient Spectrum Sharing for Noncooperative Underwater Acoustic Communication Systems
}

\author{
Antony Pottier, François-Xavier Socheleau, Christophe Laot
}

\section{To cite this version:}

Antony Pottier, François-Xavier Socheleau, Christophe Laot. Power-Efficient Spectrum Sharing for Noncooperative Underwater Acoustic Communication Systems. OCEANS 2016 - Monterey: MTS/IEEE international conference, Sep 2016, Monterey, United States. pp.1 - 6, 10.1109/OCEANS.2016.7761437 . hal-01424511

\section{HAL Id: hal-01424511 \\ https://hal.science/hal-01424511}

Submitted on 2 Jan 2017

HAL is a multi-disciplinary open access archive for the deposit and dissemination of scientific research documents, whether they are published or not. The documents may come from teaching and research institutions in France or abroad, or from public or private research centers.
L'archive ouverte pluridisciplinaire HAL, est destinée au dépôt et à la diffusion de documents scientifiques de niveau recherche, publiés ou non, émanant des établissements d'enseignement et de recherche français ou étrangers, des laboratoires publics ou privés. 


\title{
Power-Efficient Spectrum Sharing for Noncooperative Underwater Acoustic Communication Systems
}

\author{
Antony Pottier, Francois-Xavier Socheleau, Christophe Laot \\ Institut Mines-Telecom; TELECOM Bretagne, UMR CNRS 6285 Lab-STICC \\ Email: \{antony.pottier,fx.socheleau,christophe.laot\}@ telecom-bretagne.eu
}

\begin{abstract}
This paper aims at studying underwater acoustic OFDM communication systems interfering with each others in the same channel. We propose a decentralized spectrum sharing method that minimizes the total power consumed while satisfying a constraint related to their information rate. The considered systems are supposed noncooperative, i.e. unable to communicate with each others so that they cannot agree on a fair resource sharing scheme. The problem is formulated within the framework of game theory and solved according to the Nash Equilibrium concept. Several results are presented and show that interfering UA systems can share the spectrum in a more efficient way, both in terms of energy consumption and information rate.
\end{abstract}

Index Terms-Underwater acoustic communications, spectrum sharing, OFDM, interference channel, noncooperative game, power efficiency.

\section{INTRODUCTION}

Underwater acoustic (UA) communication channels feature important distance and frequency dependent attenuation that strongly limits the available communication bandwidth. This makes the UA spectrum a scarce resource potentially shared by many heterogeneous sources (communication systems, sonars, marine mammals, etc) and whose usage is not restricted by any regulatory body or standard. As a consequence, reliable UA communications are not challenging only because of the severe propagation channel, but also due to mutual interferences between several noncooperative entities wishing to use the same physical resource. This unintended jamming can drastically decrease the performance of UA systems, both in terms of information rate and energy efficiency.

Our aim in this paper is to propose a strategy able to improve the coexistence of UA communication systems transmitting in the same channel at the same time. Our interest lies in adaptive approaches, where transmitting devices are brought with the ability of automatically changing their transmission parameters according to the soundscape in which they operate.

So far, most of the works on similar topics have focused either on adaptive modulation for single user UA links [1], [2] or on cooperative multiusers UA systems evolving within the same network [3], [4]. The systems considered here are assumed to be fully noncooperative, which suppose the absence of signaling between the different communication links and the inability to agree on a resource sharing policy. In recent works [5], [6], we proposed a decentralized, adaptive power allocation strategy based on game theory for noncooperative
UA OFDM communication systems, where each transmitter seeks to maximize selfishly a criterion related to its information rate under average power constraint. It was shown that efficient power allocations over the available frequency band are attained by an iterative waterfilling solution. Numerical results have shown the benefits of this approach in high interference environments.

Here, we propose to study a situation where several interfering UA OFDM links try to minimize the total power needed to guarantee a minimum information rate. Energy consumption minimization problems are relevant for many UA applications requiring some energy autonomy or long battery life such as UA wireless networks or Autonomous Underwater Vehicles. Our contribution is to prove that UA systems can share the spectrum in an adaptive, decentralized and noncooperative manner to reach operating points where both their spectral and energy efficiencies are improved.

The paper is organized as follows: we first present the transmission and channels model, then formulate the problem of constrained total power minimization for multiple noncooperative users in Section II. A waterfilling solution based on the Nash Equilibrium concept is given in Section III. Finally, simulation results based on synthetic shallow water channels are analyzed in Section IV before conclusion in Section V.

Notations: Uppercase and lowercase boldface letters, e.g. $\mathbf{A}, \mathbf{x}$, denote matrices and vectors, respectively. The superscript ${ }^{T}$ denotes transposition. The unitary column vector is denoted by 1 . Finally, $\mathbb{E}\{$.$\} denotes expectation.$

\section{SYSTEM MODEL AND PROBLEM FORMULATION}

Based on [5], we consider $M$ UA communication links using OFDM modulation and transmitting at the same time on the same frequency band $B$ divided in $N$ subcarriers. The OFDM symbol duration and subcarrier spacing are denoted by $T$ and $\Delta f$ respectively. Considering noncooperative setups, each receiver treats the interference generated by other users as colored additive noise. Let $\mathbf{x}_{i} \in \mathbb{C}^{N}$ be the sequence of i.i.d symbols sent by transmitter $i$ on the $N$ subcarriers. For any $i$, the symbols $\mathbf{x}_{j \neq i}$ sent by the other transmitters are independent of $\mathbf{x}_{i}$. The received symbol at receiver $i$ on the $n^{\text {th }}$ subcarrier is

$$
y_{i}(n)=h_{i i}(n) x_{i}(n)+\sum_{j \neq i} h_{j i}(n) x_{j}(n)+w_{i}(n)
$$


where $w_{i}(n) \sim \mathcal{C N}\left(0, \sigma_{w_{i}}^{2}(n)\right)$ is a Gaussian noise independent of both $x_{i}(n)$ and $x_{j \neq i}(n) . h_{i i}(n) \sim \mathcal{C N}\left(\mu_{i i}(n), \sigma_{i i}^{2}(n)\right)$ is the complex gain of the direct subchannel $n$ and $h_{j i}(n) \in \mathbb{C}$ are those of the subchannels through which transmitters $j \neq i$ interfere on the receiver $i$.

In scenarios where all the links are located in the same area it may be that some (or even all) links are unreliable, due to a high interference level. Trying to establish a communication without taking into account the presence of interfering sources would lead to a waste of energy.

Our goal is to minimize the total power allocated on the whole band $B$ by each transmitter while complying to a specified information rate. As the information rate of the link $i$ will depend of the interference generated by the other links, we denote this rate by $u_{i}\left(\mathbf{p}_{i}, \mathbf{p}_{-i}\right)$ where $\mathbf{p}_{i}=\left[p_{i}(1), \cdots, p_{i}(N)\right]^{T} \in \mathbb{R}_{+}^{N}$ is the vector of powers allocated on the $N$ subcarriers by the transmitter $i$ and $\mathbf{p}_{-i}=\left[\mathbf{p}_{1}, \cdots, \mathbf{p}_{i-1}, \mathbf{p}_{i+1}, \cdots, \mathbf{p}_{M}\right]$ are all the allocation strategies chosen by the other transmitters.

Formally, we need to solve simultaneously a set of $M$ constrained optimization problems. For each individual problem, the optimization variables are the power allocations $\mathbf{p}_{i}$ of transmitters $i \in\{1, \cdots, M\}$, and optimal solutions $\mathbf{p}_{i}^{\star}$ depend on the choice of $\mathbf{p}_{-i}$ made by all others transmitters:

$$
\begin{gathered}
\mathbf{p}_{i}^{\star}=\underset{\mathbf{p}_{i}}{\operatorname{argmin}} \sum_{n=1}^{N} p_{i}(n) \\
\text { subject to }\left\{\begin{array}{l}
u_{i}\left(\mathbf{p}_{i}, \mathbf{p}_{-i}\right) \geq R_{i}^{\min } \\
p_{i}(n) \geq 0, \forall n \in\{1, \cdots, N\}
\end{array}\right.
\end{gathered}
$$

where $u_{i}\left(\mathbf{p}_{i}, \mathbf{p}_{-i}\right) \geq R_{i}^{\min }$ is the constraint on the information rate. We follow [5] to define $u_{i}$ as

$$
\begin{aligned}
& u_{i}\left(\mathbf{p}_{i}, \mathbf{p}_{-i}\right)= \\
& \frac{1}{N T \Delta f} \sum_{n=1}^{N} \log \left(1+\frac{g_{i}(n) \mathbb{E}\left\{\left|h_{i i}(n)\right|^{2}\right\} p_{i}(n)}{\sigma_{w_{i}}^{2}(n)+\sum_{j \neq i} \mathbb{E}\left\{\left|h_{j i}(n)\right|^{2}\right\} p_{j}(n)}\right),
\end{aligned}
$$

where

$$
g_{i}(n)=\frac{K_{i}(n)}{K_{i}(n)+1} e^{-\operatorname{Ei}\left(-K_{i}(n)\right)},
$$

$K_{i}(n)=\left|\mu_{i i}(n)\right|^{2} /\left|\sigma_{i i}(n)\right|^{2}$ is the Rice factor of subchannel $h_{i i}(n)$ and $\operatorname{Ei}(-x)$ denotes the exponential integral function defined, for $x>0$, as

$$
\operatorname{Ei}(-x)=-\int_{x}^{+\infty} \frac{e^{-t}}{t} d t
$$

Note that $u_{i}$ should not be understood as an achievable rate in the sense of [7] but rather as an optimization criterion allowing UA systems to find efficient power allocation strategies in a noncooperative way and with minimal knowledge about their environment. In the following, $u_{i}$ will thus be called "information rate" with a slight abuse of language.

Solving the minimization problem (1) requires that the transmitters have a knowledge only about the statistics of their direct channels $h_{i i}$ and the overall noise plus interference PSD. From a practical implementation point of view, this knowledge can be acquired via a feedback link from their respective receiver - which is supposed to estimate correctly the needed parameters. Computing these statistics on the long term can reduce the feedback activity of the receiver. It also copes with issues related to outdated channel state information (CSI) at the transmitter.

Given the structure of the problem, it is possible to reformulate it as a noncooperative game [8] defined by the following triplet:

$$
\mathcal{G}=\left\{\mathcal{M},\left\{\mathcal{P}_{i}\left(\mathbf{p}_{-i}\right)\right\}_{i=1}^{M},\left\{P_{i}^{\mathrm{tot}}\right\}_{i=1}^{M}\right\}
$$

where the set of players $\mathcal{M}=\{1, \cdots, M\}$ are the active links in the same channel, $\mathcal{P}_{i}\left(\mathbf{p}_{-i}\right)$ is the strategy space of the player $i \in \mathcal{M}$ defined as

$$
\mathcal{P}_{i}\left(\mathbf{p}_{-i}\right)=\left\{\mathbf{p}_{i} \in \mathbb{R}_{+}^{N}: u_{i}\left(\mathbf{p}_{i}, \mathbf{p}_{-i}\right) \geq R_{i}^{\text {min }}\right\}
$$

and the total transmit power $P_{i}^{\text {tot }}\left(\mathbf{p}_{i}\right)=\mathbf{1}^{T} \mathbf{p}_{i}$ is the objective function that the transmitter $i$ has to minimize.

\section{ITERATIVE WATERFILLING SOLUTION}

\section{A. Nash Equilibrium and waterfilling best-response functions}

We propose to solve the noncooperative game defined by $\mathcal{G}$ according to the concept of Nash Equilibrium (NE) [9]. In game theory, a $\mathrm{NE}$ of a noncooperative game is a strategic choice which is unilaterally optimal for each player. In the previous context, it would correspond to a set of power allocation strategies $\mathbf{p}^{\star}=\left[\mathbf{p}_{1}^{\star}, \cdots, \mathbf{p}_{M}^{\star}\right]$ such that no communication link can choose another allocation strategy $\mathbf{p}_{i} \neq \mathbf{p}_{i}^{\star}$ satisfying its information rate constraint, unless more transmit power is used. Mathematically, we have the following NE definition corresponding to the game $\mathcal{G}$ :

Definition 1: A Nash Equilibrium of the game $\mathcal{G}$ as defined in (3) is a strategy profile $\mathbf{p}^{\star}=\left[\mathbf{p}_{1}^{\star}, \cdots, \mathbf{p}_{M}^{\star}\right]$ such that $\forall i \in$ $\mathcal{M}$ and $\forall \mathbf{p}_{i} \in \mathcal{P}_{i}\left(\mathbf{p}_{-i}^{\star}\right)$ we have

$$
\sum_{n=1}^{N} p_{i}^{\star}(n) \leq \sum_{n=1}^{N} p_{i}(n)
$$

In order to drive our systems to a Nash Equilibrium point of the game, we need to define their best response functions [8] :

Definition 2: Given a fixed strategic choice $\mathbf{p}_{-i} \in \mathbb{R}_{+}^{N}$ of his opponents, the best response function of the player $i$ to $\mathbf{p}_{-i}$ is the set of strategies $\mathbf{p}_{i}^{\star}$ such that

$$
\sum_{n=1}^{N} p_{i}^{\star}(n) \leq \sum_{n=1}^{N} p_{i}(n), \forall \mathbf{p}_{i} \in \mathcal{P}_{i}\left(\mathbf{p}_{i}\right) .
$$

In other words, the best response function of a player $i$ corresponds to the unique solution of (1) for a fixed nonnegative $\mathbf{p}_{-i}[10]$ and can be found by solving the Karush-Kuhn-Tucker system of equations associated to the dual problem of (1). This yields the waterfilling solution $\forall i \in \mathcal{M}$ and $\forall n=1, \cdots, N$

$$
p_{i}^{\star}(n)=\left[\frac{1}{\lambda_{i}}-\frac{\sigma_{w_{i}}^{2}(n)+\sum_{j \neq i} \mathbb{E}\left\{\left|h_{j i}(n)\right|^{2}\right\} p_{j}(n)}{g_{i}(n) \mathbb{E}\left\{\left|h_{i i}(n)\right|^{2}\right\}}\right]^{+},
$$

where $[x]^{+}$is equivalent to $\max (0, x)$ and $\lambda_{i}$ is chosen to satisfy the information rate constraint $u_{i}\left(\mathbf{p}_{i}, \mathbf{p}_{-i}\right) \geq R_{i}^{\text {min }}$ with equality. 
Then, combining Definitions 1 and 2, it appears that a NE of the game $\mathcal{G}$ in (3) is reached when all the communication links use the waterfilling strategy (7) to allocate their power among the $N$ subcarriers. In practice, the NEs of $\mathcal{G}$ have to be reached iteratively [10], [11]. In this paper we choose to focus on a distributed sequential waterfilling algorithm where the communication links update their strategy one after the other, one link being chosen at each iteration. Questions that can arise are wether or not a bounded power allocation solution exists for a given set of channels and rate constraints and if it can be reached iteratively.

\section{B. Existence of a solution and convergence of iterative algo- rithms}

In general, existence of at least one NE is guaranteed for any game whose objective functions are concave and strategy spaces are closed and bounded convex sets [13]. However, given the problem stated in (1), the strategy spaces are not bounded since there is no constraints on the maximum total power. As a consequence, it is possible that the set of channels $h_{j i}$ and the rate constraints lead to a situation where some communication links have no other choice than increasing their transmit power each time they have to update their power allocation strategy in order to maintain their information rate constraint satisfied. Sufficient conditions on the existence of a non-empty, bounded set of solutions for the game $\mathcal{G}$ in (3) have been given in [10, Theorem 5] and will be adapted next to our context. We first need to introduce the matrices $\boldsymbol{\Psi}_{n}\left(\mathbf{R}^{\text {min }}\right) \in \mathbb{R}^{M \times M}$, given a specified set of rate constraints $\mathbf{R}^{\text {min }}=\left[R_{1}^{\min }, \cdots, R_{M}^{\min }\right]^{T}>\mathbf{0}$ :

$$
\left[\boldsymbol{\Psi}_{n}\left(\mathbf{R}^{\text {min }}\right)\right]_{i j} \triangleq\left\{\begin{aligned}
g_{i}(n) \mathbb{E}\left\{\left|h_{i i}(n)\right|^{2}\right\} & \text { if } i=j \\
-\left(e^{R_{j}^{m i n}}-1\right) \mathbb{E}\left\{\left|h_{j i}(n)\right|^{2}\right\} & \text { otherwise }
\end{aligned}\right.
$$

Theorem 1: [10, Theorem 5] The game $\mathcal{G}$ with rate constraints $\mathbf{R}^{\text {min }}=\left[R_{1}^{\text {min }}, \cdots, R_{M}^{\text {min }}\right]^{T}>\mathbf{0}$ has a non-empty and bounded solution set if $\boldsymbol{\Psi}_{n}\left(\mathbf{R}^{\text {min }}\right)$ in (8) are such that all their principal minors are positive i.e.

$$
\left|\mathbf{C}_{\Psi_{n}}^{(i, j)}\right| \geq 0, \forall n=1, \cdots, N
$$

with $\left|\mathbf{C}_{\mathbf{\Psi}_{n}}^{(i, j)}\right|$ the determinant of the cofactor matrix obtained by removing the $i^{\text {th }}$ raw and $j^{\text {th }}$ column from $\Psi_{n}\left(\mathbf{R}^{\text {min }}\right)$. Moreover, an upper bound $\overline{\mathbf{p}}=\left[\overline{\mathbf{p}}_{1}, \cdots, \overline{\mathbf{p}}_{M}\right]$ on the power allocation vectors at a NE can be given $\forall n=1, \cdots, N$ as

$$
\left[\begin{array}{c}
\overline{\mathbf{p}}_{1}(n) \\
\vdots \\
\overline{\mathbf{p}}_{M}(n)
\end{array}\right]=\left(\boldsymbol{\Psi}_{n}\left(\mathbf{R}^{m i n}\right)\right)^{-1}\left[\begin{array}{c}
\sigma_{w_{1}}^{2}(n)\left(e^{R_{1}^{m i n}}-1\right) \\
\vdots \\
\sigma_{w_{M}}^{2}(n)\left(e^{R_{M}^{m i n}}-1\right) .
\end{array}\right]
$$

Interestingly, one can verify that these conditions always hold in the two user case.

Corollary 1: Sufficient conditions for the matrices $\boldsymbol{\Psi}_{n}\left(\mathbf{R}^{\text {min }}\right)$ to have all their principal minors positive are :

$$
R_{i}^{\text {min }}<\log \left(\frac{1}{\gamma_{i}^{\text {max }}}+1\right), \forall i \in \mathcal{M}
$$

with

$$
\gamma_{i}^{\max } \triangleq \max _{n} \sum_{j \neq i} \frac{\mathbb{E}\left\{\left|h_{j i}(n)\right|^{2}\right\}}{g_{i}(n) \mathbb{E}\left\{\left|h_{i i}(n)\right|^{2}\right\}} .
$$

The previous corollary is useful to derive a simple physical interpretation of Theorem 1: as the interferences become higher, having a bounded solution to the problem (1) requires that the rate constraints imposed on each communication link are not too high.

Usually, convergence of iterative best-response algorithms, the sequential iterative waterfilling being one example, is guaranteed only in games with some particular properties. In most of other communication games, convergence proofs are application-specific and only sufficient conditions can be given [11]. We focus on a sequential version of the iterative waterfilling algorithm, for which convergence conditions can be derived [10, Theorem 14]. Let first $\boldsymbol{\Xi}_{n}\left(\mathbf{R}^{\text {min }}\right) \in \mathbb{R}^{M \times M}$ be

$$
\left[\boldsymbol{\Xi}_{n}\left(\mathbf{R}^{\min }\right)\right]_{i j} \triangleq\left\{\begin{array}{cl}
e^{-R_{i}^{\text {min }}} & \text { if } i=j \\
-e^{R_{j}^{\text {min }}} \xi_{i j}^{\max } & \text { otherwise }
\end{array}\right.
$$

where

$$
\begin{aligned}
\xi_{i j}^{\max } \triangleq & \max _{n} \frac{\mathbb{E}\left\{\left|h_{j i}(n)\right|^{2}\right\}}{g_{j}(n) \mathbb{E}\left\{\left|h_{j j}(n)\right|^{2}\right\}} \\
& \times \frac{\sigma_{w_{j}}^{2}(n)+\sum_{k \neq j} \mathbb{E}\left\{\left|h_{k j}(n)\right|^{2}\right\} \bar{p}_{k}(n)}{\sigma_{w_{i}}^{2}(n)}
\end{aligned}
$$

and $\bar{p}_{k}(n)$ defined as in (9).

Theorem 2: [10, Theorem 14] Assume that the conditions of Theorem 1 are satisfied for the game $\mathcal{G}$ with rate constraints $\mathbf{R}^{\text {min }}$. If the matrix $\boldsymbol{\Xi}_{n}\left(\mathbf{R}^{\text {min }}\right)$ as defined in (11) has its principal minors all positive, then the sequential waterfilling algorithm converges to the (unique) NE of the game.

Note that the previous theorem gives also sufficient conditions for uniqueness of the NE of the game $\mathcal{G}$. Furthermore, it can also be interpreted as a requirement of sufficiently small interference level perceived by each communication link [10, Remark 15].

The conditions of Theorems (1) and (2) can be hard to check and may be unsatisfied in many scenarios of practical interest where one can expect that good operating points can be reached by iterative waterfilling (see for instance [5], [6]). Indeed, as these two theorems give sufficient conditions on solutions boundedness and algorithms convergence, it does not imply that their non-satisfaction will lead necessarily to divergence and unbounded power budget. Nevertheless, they are useful to warn us that if the level of interferences and/or the desired information rates are too high, the whole system cannot reach an equilibrium.

\section{NumERICAL RESUlTS}

We here show some simulation results for three noncooperative UA OFDM links with $N=256$ subcarriers in the same bandwidth $B=6 \mathrm{kHz}$ centered around $f_{c}=12 \mathrm{kHz}$. The subcarrier spacing and OFDM symbol time are $\Delta f=23.4 \mathrm{~Hz}$ and $T=57.7 \mathrm{~ms}$, including a $15 \mathrm{~ms}$ cyclic prefix time. Direct and cross channels $h_{j i}, i, j \in\{1,2,3\}$, are generated by an 

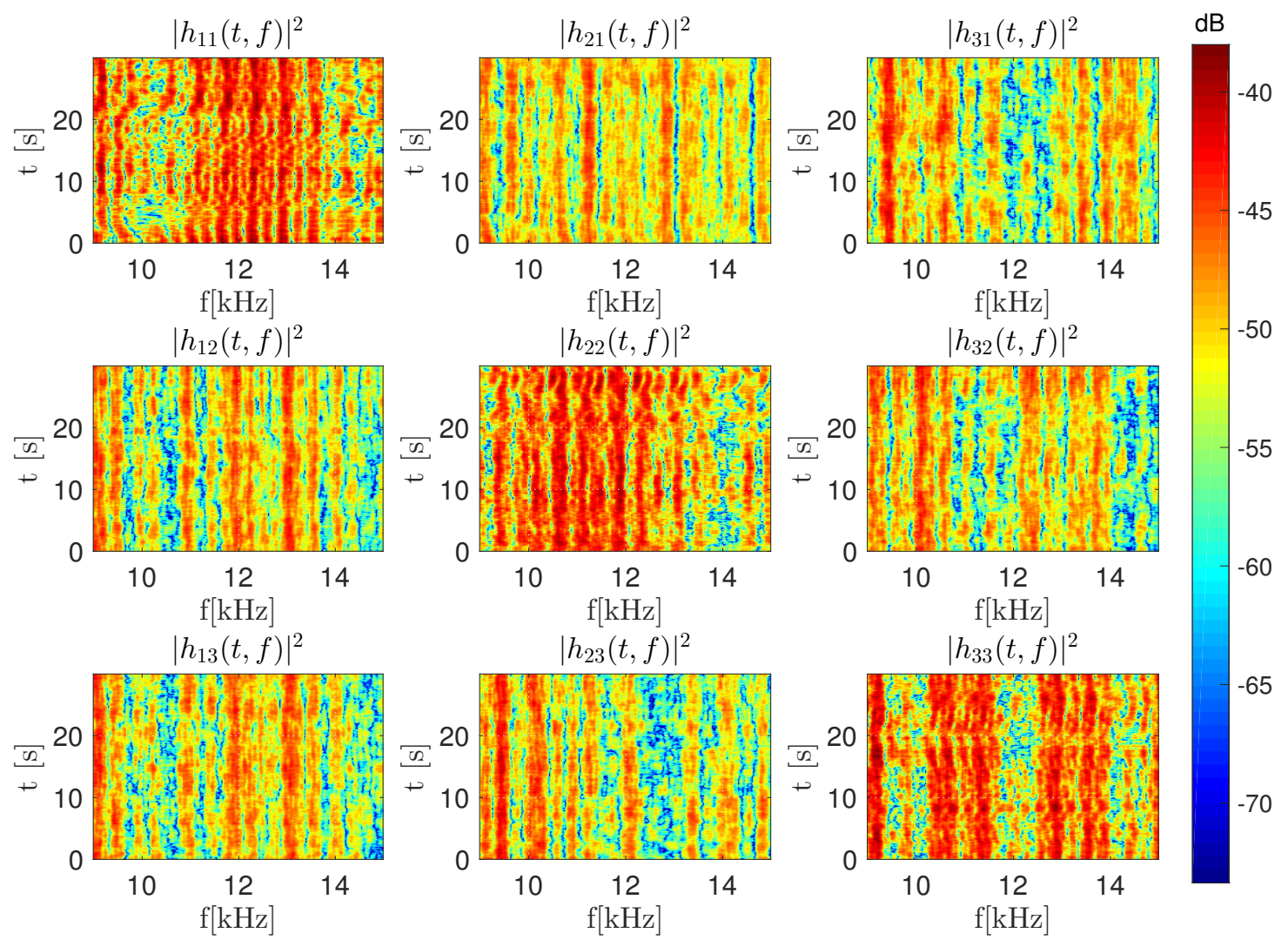

Fig. 1. Time-varying frequency responses of the directs and interference channels obtained from the simulator described in [12]. $d_{i i}=1 \mathrm{~km}, d_{j i}=1.5 \mathrm{~km}$.

UA shallow water channel simulator described in [12], [14]. The impulse responses are obtained by ray tracing according to imput parameters describing the transmission geometry. Time fluctuations are then modeled by a maximum entropy Doppler spectrum given the mean Doppler spread $\sigma_{D}$ and Rice factor $K^{\max }$ of the main arrival. The Rice factor of other arrivals decreases exponentially. In the following, we set $\sigma_{D}=1 \mathrm{~Hz}$ and $K^{\max }=20 \mathrm{~dB}$. The water depth is fixed to $50 \mathrm{~m}$ and the depth of each terminal is chosen arbitrarily between 5 and $20 \mathrm{~m}$. Path losses and frequency-dependent absorption are taken into account using Thorp's formula [15]. The distances $d_{i i}$ between a transmitter and its intended receiver are set to $1 \mathrm{~km}$ and we set their initial total transmit power to 160 $\mathrm{dB}$ re $\mu \mathrm{Pa}$, distributed uniformly on the whole $6 \mathrm{kHz}$ band. The noise PSD is modeled with a decay of $18 \mathrm{~dB} /$ decade and its power in the frequency band in use is such that the communication links experiences a signal to noise ratio (SNR) of $15 \mathrm{~dB}$ for their initial uniform power allocation (without considering interferences). Each receiver is supposed to estimate its direct channel statistics $\mu_{i i}(n)$ and $\sigma_{i i}^{2}(n)$ as well as the noise plus interference PSD without errors. These parameters are used by the corresponding transmitter to find the power allocation solving the problem (1), with the constraint $u_{i}\left(\mathbf{p}_{i}, \mathbf{p}_{-i}\right)=R_{i}^{\min }$. The update period of each link is set to 30 seconds, which is long enough compared to the OFDM symbol duration and channel coherence time to assume ergodicity. The links update their power allocation strategy one after the other ${ }^{1}$ and each update (from any player) is called "an iteration" of the game in the following. The game is run during 50 iterations.

We will consider several scenarios by varying the interference links distances $d_{j i}$ and the rate constraints.

\section{A. Medium interference scenario}

The distances of the interference links are set to $d_{j i}=1.5$ $\mathrm{km}$ and the rate constraints are $u_{i}\left(\mathbf{p}_{i}, \mathbf{p}_{-i}\right)=1$ bits $/ \mathrm{s} / \mathrm{Hz}$ for all the links. The time varying frequency responses of the channels for this setup are shown in Figure 1, where the diagonal shows what we call the direct channels $h_{i i}$ between a transmitter $i$ and its intended receiver, and the other subplots depicts the interference channels $h_{j i}$ between the $j^{\text {th }}$ transmitter and the $i^{\text {th }}$ receiver. Here, we noted that the conditions required by Theorem 1 are not met.

Figure 2 depicts the total power in use by the three communication links as a function of iterations of the game. After 15 iterations - corresponding to 2.5 minutes given the update period - the users are close to a state where none of them has an incentive to decrease or increase its transmit power to satisfy the information rate constraint. The evolution of the information rate metric $u_{i}(2)$ is depicted in Figure 3. In dashed lines, we compare these results with the initial uniform power strategy with the total power of $160 \mathrm{~dB}$ re $\mu \mathrm{Pa}$

\footnotetext{
${ }^{1}$ Note that it is not necessary to assume synchronism among the links, since we can consider systems having the same update period but starting to transmit at different times.
} 


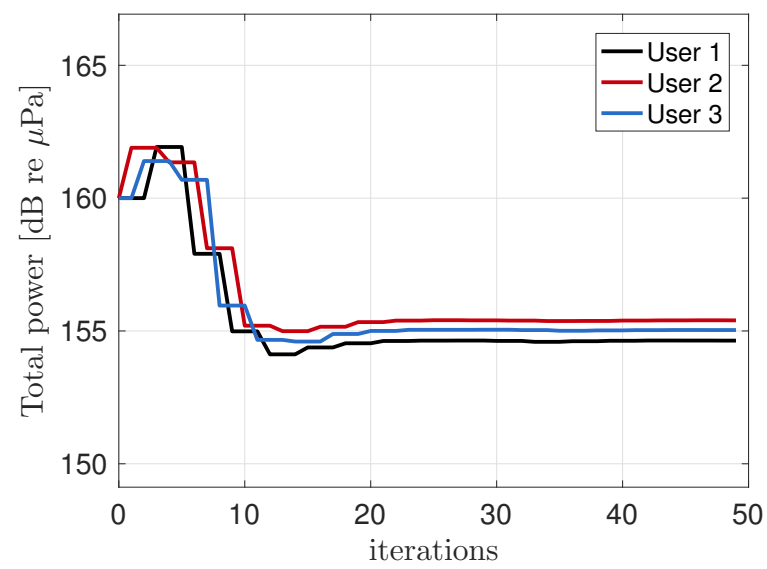

Fig. 2. Total power in use versus iterations of the game (medium interference).

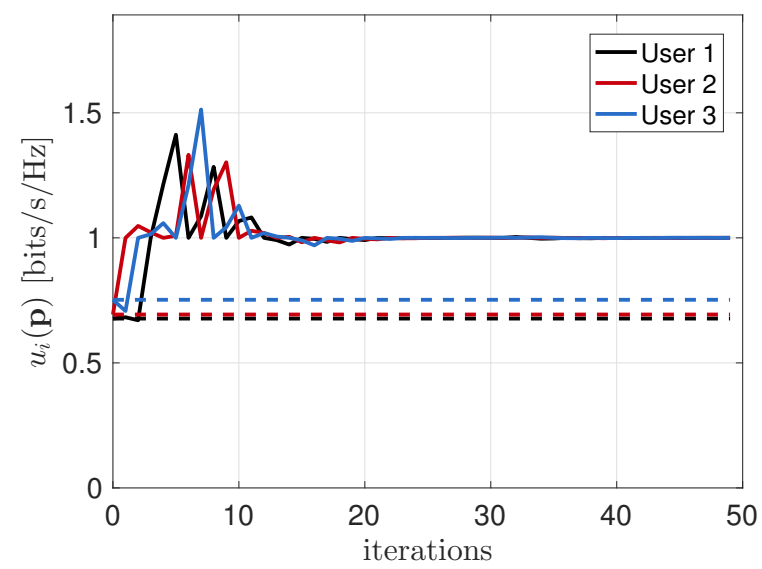

Fig. 3. Information rate function versus iterations of the game (medium interference).

(denoted by $P_{i}^{I N I T}$ in Figure 2). A clear improvement both in terms of spectral efficiency and total power consumption can be observed since our proposal of noncooperative and adaptive spectrum sharing strategy yields a better information rate metric $u_{i}$ with only $30 \%$ of the initial total power (approximately $5 \mathrm{~dB}$ less).

\section{B. High interference scenario}

In this scenario we decrease the distance separating the interfering links to simulate a high interference setup. The distances $d_{j i}$ are set to $500 \mathrm{~m}$, so that at the initial uniform power allocation state, each link perceives approximately twice more interference than signal of interest. ${ }^{2}$ The results of the noncooperative game after 50 iterations in terms of total power and information rate functions are shown in Figures 4 and 5 respectively.

After 25 iterations the three communications links are in an equilibrium state. Most importantly we see that they have considerably increase their information rate function $u_{i}$ compared to the initial state (in dashed lines), and with a decrease of total power in use of more than $5 \mathrm{~dB}$. This is

\footnotetext{
${ }^{2}$ In a real setup this would obviously impact the performances of the channel estimations required to solve the minimization problem (1).
}

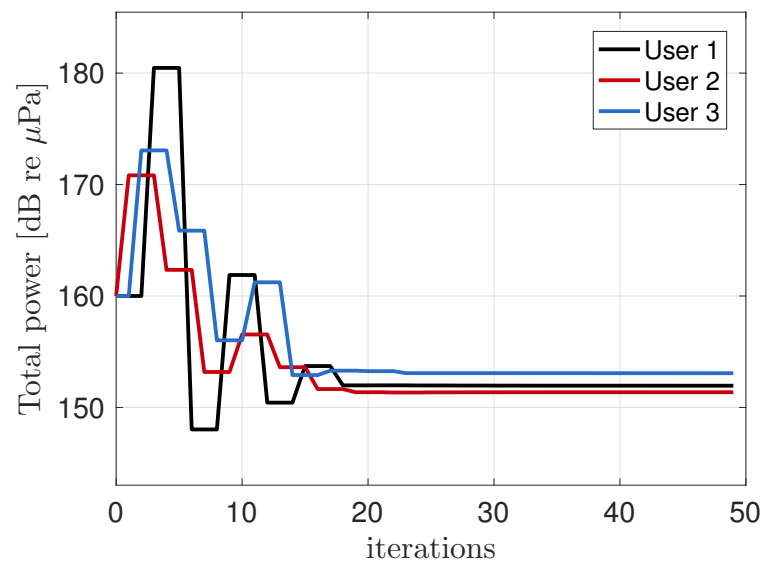

Fig. 4. Total power in use versus iterations of the game (high interference).

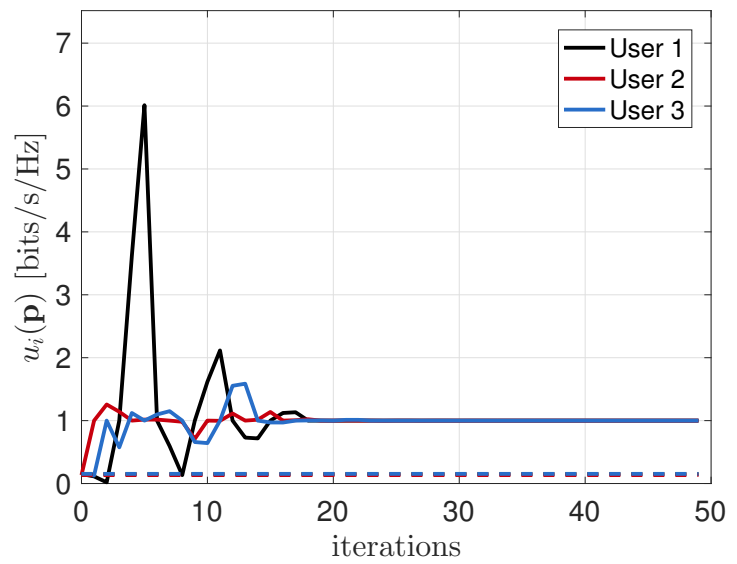

Fig. 5. Information rate function versus iterations of the game (high interference).

due to the fact that as the interference level is high, the best strategic choice for the players tends to a FDMA-like power allocation as the Figure 6 depicts. This fact has been pointed out in [16], [17].

However, it can be seen from Figure 4 that before reaching the equilibrium state, all links need to increase considerably their total transmit power during the convergence phase. Depending on the initial power, such an increase could be prohibitive for many UA systems. During the first iterations, each player increases its total power one after the other and releases or decreases its power on some subcarriers at the same time. They act this way repeatedly until each of them have succeed in pushing the others out of its subcarriers of most interest, leading to a strategy profile close to FDMA. Then, the next iterations lead them to reduce the power allocated where they experience still some interference and increase it on their good subcarriers, until the information rate constraints are simultaneously satisfied. Interestingly, it has been proven that the sum-rate optimal power allocation is an FDMAtype strategy when the crosstalk between the different links is sufficiently high [18]. In the previously cited reference, a locally optimal FDMA power allocation is found by means of a centralized algorithm, assuming the knowledge of all the direct and interference channels. In contrast, our present work 


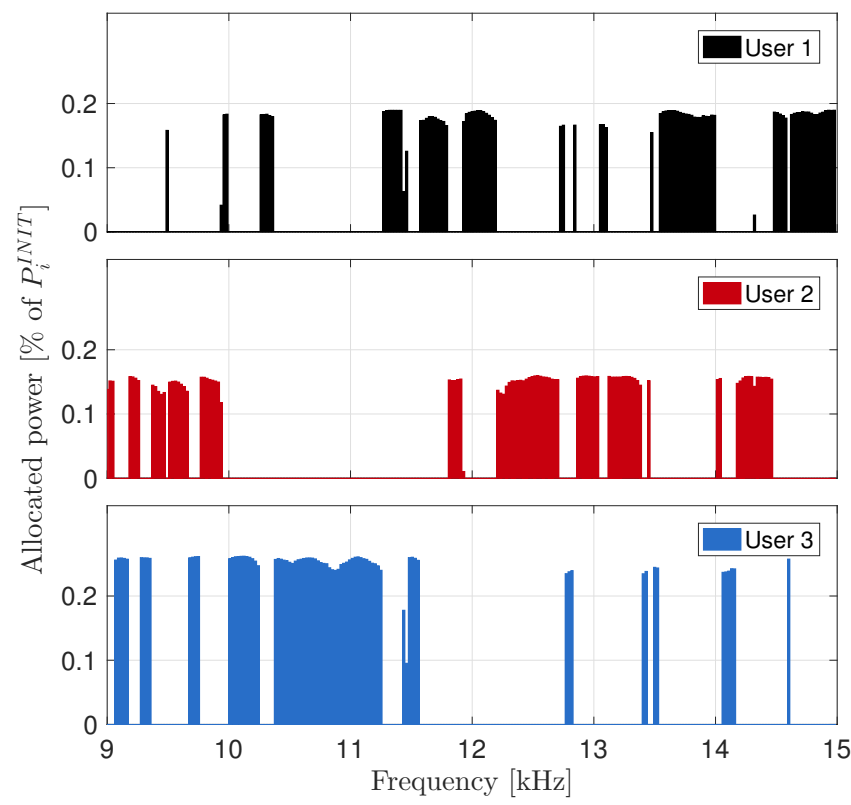

Fig. 6. Power allocated by the users at the last iteration of the game (in \% of the initial power $P_{i}^{I N I T}=160 \mathrm{~dB}$ re $\mu \mathrm{Pa}$ ).

assume only little knowledge of the environment from the communication links involved in the problem - namely, only the direct channels statistics $\left(h_{i i}\right)$ and noise plus aggregate interference PSD.

\section{CONClusion}

The proposed distributed power allocation strategy allows several interfering UA OFDM communication links to share the spectrum in an efficient way regarding the total transmit power in use, compared to the naive uniform transmit PSD strategy which leads to waste of energy in the studied scenarios. Our method has been developed for fully noncooperative systems competing for the same physical resource. Moreover, it requires only a little knowledge on the ambient soundscape by the transmitters to adapt their power allocation strategy. The main drawback of this method relies on the fact that convergence of distributed algorithms used to reach the Nash Equilibrium solutions is not guaranteed in many scenarios of practical interest, where the interference level is high. In addition, situations where an equilibrium state is reached at the cost of a considerable increase of the power budget have been observed. To be closer from practical UA systems requirements, future works on the topic could consider a modified game, with for instance an additional constraint on the maximum average power or systems having the ability to adapt their rate constraints automatically when the use of too high powers is observed.

\section{REFERENCES}

[1] A. Radosevic, R. Ahmed, T.M. Duman, J. G. Proakis, and M. Stojanovic, "Adaptive OFDM Modulation for Underwater Acoustic Communications: Design Considerations and Experimental Results," IEEE J. Ocean. Eng., vol. 39, no. 2, pp. 357-370, Apr 2014

[2] Y. Aval, S. K. Wilson, and M. Stojanovic, "On the Achievable Rate of a Class of Acoustic Channels and Practical Power Allocation Strategies for OFDM Systems," IEEE J. Ocean. Eng., vol. 40, no. 4, pp. 785-795, Oct 2015 .
[3] P. Wang, X. Zhang, and M. Song, "Power-efficient Resource Allocation for QoS Provisioning in Underwater MIMO-OFDM Acoustic Cooperative Wireless Networks," in Global Communications Conference (GLOBECOM), 2013 IEEE, Dec 2013, pp. 4674-4678.

[4] R. Otnes, A. Asterjadhi, P. Casari, M. Goetz, T. Husoy, I. Nissen, K. Rimstad, P. van Walree, and M. Zorzi, Underwater Acoustic Networking Techniques, Springer Briefs in Electrical and Computer Engineering, 2012.

[5] A. Pottier, F.-X. Socheleau, and C. Laot, "Distributed Power Allocation Strategy in Shallow Water Acoustic Interference Channels," in IEEE Int. workshop on Signal Processing Advances in Wireless Communications (SPAWC) 2016, Jul. 2016.

[6] A. Pottier, F.-X. Socheleau, and C. Laot, "Adaptive Power Allocation for Noncooperative OFDM Systems in UWA Interference Channels," in Underwater Acoustic Communications and Networking (UComms) 2016 2016, Sept. 2016.

[7] J.-M. Passerieux, F.-X. Socheleau, and C. Laot, "Achievable Rates over Doubly Selective Rician-Fading Channels under Peak-Power Constraint," IEEE Trans. Wireless Commun., vol. 12, no. 2, pp. 586-594, February 2013.

[8] Z. Han, D. Niyato, W. Saad, T. Basar, and A. Hjorungnes, Game Theory in Wireless and Communication Networks, Cambridge University Press, 2012.

[9] J. F. Nash, "Non Cooperative Games," The Annals of Mathematics, vol. 54, pp. 286-295, September 1951.

[10] J. S. Pang, G. Scutari, F. Facchinei, and C. Wang, "Distributed Power Allocation With Rate Constraints in Gaussian Parallel Interference Channels," IEEE Transactions on Information Theory, vol. 54, no. 8, pp. 3471-3489, Aug 2008 .

[11] G. Bacci, S. Lasaulce, W. Saad, and L. Sanguinetti, "Game Theory for Networks: A tutorial on game-theoretic tools for emerging signal processing applications," IEEE Signal Processing Magazine, vol. 33, no. 1, pp. 94-119, January 2016.

[12] F-X. Socheleau, C. Laot, and Passerieux J-M., "A Maximum Entropy Framework for Statistical Modeling of Underwater Acoustic Communication Channels," in MTS/IEEE Oceans 2010, Sydney, June 2010, pp. $1-7$.

[13] J. B. Rosen, "Existence and Uniqueness of Equilibrium Points for Concave N-Persons Games," Econometrica, vol. 33, no. 3, pp. 521534, July 1965

[14] F-X. Socheleau, C. Laot, and J-M. Passerieux, "Concise Derivation of Scattering Function from Channel Entropy Maximization," IEEE Trans. on Communications, vol. 58, no. 11, pp. 3098-3103, November 2010.

[15] R. J. Urick, Principles of Underwater Sound, 3rd Edition, McGraw-Hill, 1983.

[16] A. Lesherm and E. Zehavi, "Game Theory and the Frequency Selective Interference Channel," IEEE Signal Processing Mag., vol. 26, pp. 28-40, September 2009.

[17] G. Scutari, D. P. Palomar, and S. Barbarossa, "Optimal Linear Precoding Strategies for Wideband Non-Cooperative Systems Based on Game Theory-Part I: Nash Equilibria," IEEE Trans. Signal Process., vol. 56, no. 3, pp. 1230-1249, March 2008.

[18] S. Hayashi and Z.Q Luo, "Spectrum Management for InterferenceLimited Multiuser Communication Systems," IEEE Trans. Information Theory, vol. 55, no. 3, pp. 1153-1175, March 2009. 